\title{
Editorial: science and engineering, two aspects of materials
}

\section{Editorial}

Materials Science and Engineering are clearly represented in the published articles in the journal MSEIJ this year. Materials science are focusing on the new materials in nano scales instead of macro scale or mega structure, in forms of $1 \mathrm{D}$ or $2 \mathrm{D}$ such as membranes and fibers instead of massive blocks, in functions instead of structure, in catalyst instead of the reaction agents themselves, in the application in biology, heath, climate instead of machine, equipment's and buildings. On the other hand, material engineering covers the traditional materials mainly in the application of macro scale and mega structures such as roads, dams, plants. However, the effect of industry on environment and how to realize sustainable development is also a hot topic Air quality is vital to our heath. Thus, sensors to detect harmful gases, toxic gases, unpleasant odors, volatile organic compounds (VOCs) and air pollutants are very important. In one article screen printed electrodes (SPEs) for gas sensors which are based on polymer/carbon nanostructures (multi wall carbon nanotubes and grapheme) were modified by polymers such as conductive polymer Poly Vinylidene Fluoride and biocompatible Poly Ethylene Glycol. The modified sensor electrodes exhibited excellent electrical properties, characterized by high conductivity and great potential application in the field of gas pollutants detection. In another article, Graphite oxide $(\mathrm{GO})$ was used to anchor $\left[\mathrm{PdCl}_{2}(\mathrm{TDA})_{2}\right]$ and $\left[\mathrm{RhCl}(\mathrm{TDA})_{3}\right]\left(\mathrm{TDA}=\mathrm{NH}_{2}\left(\mathrm{CH}_{2}\right)_{12} \mathrm{CH}_{3}\right)$ with $2 \mathrm{wt} \%$ of metal load. The test results showed that [RhCl(TDA) $\left.)_{3}\right]$ GO was the best catalyst due to electronic and steric effects as well as the influence of the support.

A modified glassy micro-particles glucose biosensor was developed applying a new and effective procedure to anchor glucose oxidase on glassy micro particles using urea medium. The resulting sensor, in the presence of glucose, generated a color development in 60 seconds working at room temperature. The greenhouse effect has caused great influence on climate. The greenhouse gas is the release of massive amounts of carbon products into the atmosphere. In an opinion article, nanomaterials for carbon capture and conversion were discussed. It is pointed out that the development of nanostructured catalysts (or nanocatalysts) with enhanced functionality and multifunctional nanomaterials is one potentially promising field of research. Metallurgy is a main part of modern industry. The production of steels is approaching around two billons tons in the whole world. It has brought great effect on the environment. In recent years, the situation has been greatly improved because attention is paid and great effects and measures are taken and the great progress of metallurgy technology is achieved. Here an article discussed the effect of an iron and steel work in Nigeria on the effect of the River Niger. The steel company uses large quantities of water for processing various products and therefore tends to cause effluent when disposed into the river. The effect of the generated effluents on public health, safety, and the surrounding environments was investigated.

The filter of an embankment dam has to be designed to satisfy strict filtering and drainage properties. Nonetheless, segregation, which develops in homogeneities by separating the coarser fraction from the finer matrix, is by practical experience difficult to avoid. A paper
Volume 5 Issue I - 202I

\author{
Kang Jinwu \\ School of materials science and Engineering,Tsinghua University, \\ China
}

Correspondence: Kang Jinwu, School of materials Science and Engineering, Tsinghua University, Beijing, China, Fax +861062773637, Tel +8610-62784537, Email kangjw@tsinghua.edu.cn

Received: January 04, 2021 | Published: January 06, 2021

investigated the segregation phenomena and concluded that the filter material in a dry state segregates the most, but as the water content increases the segregation is reduced significantly as it nears optimized water content; however, acceptable resistance to segregation is achieved at about $30 \%$ of the optimized water level. Pavement performance is a key parameter that governs the serviceability of road networks. In an article, various mechanistic analyses of the base layer treated with various stabilizing agents were performed using the 3-D Move Analysis software to study their effect on rutting and fatigue resistance performance. The analysis showed that the use of stabilizing agents increased the pavement performance up to $96 \%$ for fatigue cracking and $34.4 \%$ for rutting. It is essential to use natural, nonpolluting and low-cost reagents in froth flotation in order to achieve a more sustainable and environmentally friendly process in mineral industry. In an article, flotation tests were carried out with high purity apatite crystals using a mixture of a Brazilian fruit (Macaúba) and seed oil (Jatropha Curcas). It is found that the Macaúba pulp's oil, highly enriched in oleic acid (omega-9), had an expressive capacity to adsorb and hydrophilizing the apatite surface. Optical properties of semiconductors play a critical role in various applications including the design and manufacture of optical components, devices \& sources, energy conversion and process monitoring \& control. The optical properties of Silicon on Insulator (SOI) and Ge photodetectors, based on Forouhi-Bloomer dispersion equation, as function of photon energy (or wavelength) and thickness are numerically simulated in an article. The results are helpful for the determination and realization of the optical response of materials under conditions of varying photon energy and thickness. As the research on materials is getting more and more intensive, great progresses are in anticipation, which will further bring revolution to our life and society.

\section{Acknowledgements}

None.

\section{Conflicts of interest}

The author declares that there is no conflict of interest. 